1 Running title (optional): Choosing the optimal population for GWAS

3 Core ideas (3-5 impact statements, 85 char max for each)

4 - Genome-wide association studies with mixture populations are expected to improve the

5 detection power of novel genes due to the increase of the sample size although the influence of

6 population structure is a concern.

7 - When a quantitative trait nucleotide (QTN) is polymorphic in a target population, a

8 combination of the target population and a population with higher diversity than the target

9 population improves the detection power of the QTN.

10 - We found that the fixation index $\left(F_{S T}\right)$ and the expected heterozygosity $\left(H_{e}\right)$ were strongly

11 related to the detection power of QTNs.

12 - Germplasm collections which have been already sequenced/genotyped are useful for improving

13 the detection power of GWAS without any addition of sequence costs by using a subset of them

14 with a target population. 


\section{Choosing the optimal population for a genome-wide association study: a simulation using} whole-genome sequences from rice

Kosuke Hamazaki, Hiromi Kajiya-Kanegae, Masanori Yamasaki, Kaworu Ebana, Shiori Yabe, Hiroshi Nakagawa and Hiroyoshi Iwata*

Affiliations:

K. Hamazaki, H. Kajiya-Kanegae and H. Iwata, Department of Agricultural and Environmental Biology, Graduate School of Agricultural and Life Sciences, The University of Tokyo, 1-1-1 Yayoi, Bunkyo-ku, Tokyo 113-8657, Japan; H. Kajiya-Kanegae, current address: Research Center for Agricultural Information Technology, National Agriculture and Food Research Organization, 3-1-1 Kannondai, Tsukuba, Ibaraki 305-8517, Japan; M. Yamasaki, Food Resources Education and Research Center, Graduate School of Agricultural Science, Kobe University, 1348 Uzurano, Kasai, Hyogo 675-2103, Japan; K. Ebana, Genetic Resources Center, National Agriculture and Food Research Organization, 2-1-2 Kannondai, Tsukuba, Ibaraki 3058602, Japan; S. Yabe, Institute of Crop Science, National Agriculture and Food Research Organization, 2-1-2 Kannondai, Tsukuba, Ibaraki 305-8518, Japan; H. Nakagawa, Institute for Agro-Environmental Sciences, National Agriculture and Food Research Organization, 3-1-3 Kannondai, Tsukuba, Ibaraki 305-8604, Japan. *Corresponding author (aiwata@mail.ecc.utokyo.ac.jp) 
37 AUC, area under the curve; CDR, correct detection rate; FDR, false discovery rate; FN, false negative FP, false positive; $F_{S T}$, the fixation index; GWAS, genome-wide association study; $\mathrm{H}$, high; HM, higher-middle; $H_{e}$, the expected heterozygosity; L, low; LD, linkage disequilibrium; LM, lower-middle; M, middle MAF, minor allele frequency QTL, quantitative trait loci; QTN, quantitative trait nucleotide; ROC, receiver operating characteristic; SNP, single nucleotide polymorphism; TN, true negative; $\mathrm{TP}$, true positive.

ABSTRACT

A genome-wide association study (GWAS) needs to have a suitable population. The factors that affect a GWAS, e.g. population structure, sample size, and sequence analysis and field testing costs, need to be considered. Mixture populations containing subpopulations of different genetic backgrounds may be suitable populations. We conducted simulation experiments to see if a population with high genetic diversity, e.g., a diversity panel, should be added to a target population, especially when the target population harbors small genetic diversity. The target population was 112 accessions of Oryza sativa subsp. japonica, mainly developed in Japan. We combined the target population with three populations that had higher genetic diversities. These were 100 indica accessions, 100 japonica accessions, and 100 accessions with various genetic backgrounds. The results showed that the GWAS power with a mixture population was generally higher than with a separate population. Also, the GWAS optimal population varied depending on the fixation index $F_{S T}$ of the quantitative trait nucleotide (QTN) and its polymorphism of QTN in each population. When a QTN is polymorphic in a target population, a target population combined with a higher diversity population improves the 
59 QTN detection power. Investigating $F_{S T}$ and the expected heterozygosity $H_{e}$ as factors

60

61

62

63

64

65

66

67

68

69

70

71

72

73

74

75

76

77

78

79

80

influencing the detection power, we showed that SNPs with high $F_{S T}$ or low $H_{e}$ are less likely to be detected by GWAS with mixture populations. Sequenced/genotyped germplasm collections can improve the GWAS detection power by using a subset of them with a target population.

\section{INTRODUCTION}

Recently, as genome sequencing costs have continued to decrease (Metzker, 2010), the whole-genome sequences of a large number of cultivars/lines have become available for major crop species, such as rice (Li et al., 2014; Wang et al., 2018). A genome-wide association study (GWAS) based on whole-genome sequences can more efficiently and accurately identify genes that control important agronomic traits than previous methods (Koboldt et al., 2013; Ott et al., 2015; Yano et al., 2016).

It is important to prepare an appropriate population to be analyzed when attempting to detect candidate genes using GWAS techniques. For example, to avoid potential false positives caused by population stratification/structure, a GWAS population should be selected that results in low stratification (Begum et al., 2015; Yano et al., 2016). However, if such a population is selected as an analytical population for a GWAS, the sample size may be limited and the detection power of the GWAS will decrease (Korte and Farlow, 2013). Therefore, when designing an appropriate GWAS population, one should be aware of the trade-off relationship between population stratification and sample size.

When preparing the population to be analyzed, the factors that directly affect the GWAS results, such as population structure, sample size, and the sequence analysis and cultivation 
81 testing costs, need to be considered. In recent years, the whole-genome sequences of a large

82

83

number of cultivars/lines have become publicly available due to highly efficient sequencing analyses and database enrichment. The publically available whole-genome sequence data will improve GWASs and could enable researchers to avoid the costs of sequencing analyses. For example, in rice, "The 3,000 Rice Genomes Project" (Li et al., 2014; Wang et al., 2018) by the International Rice Research Institute (IRRI) is a well-known whole-genome sequence dataset that is available in the "Rice SNP-Seek Database" (Alexandrov et al., 2015; Mansueto et al., 2016; 2017). Therefore, an appropriate GWAS population could potentially utilize existing public sequence data.

A mixture population obtained by mixing subpopulations with different genetic backgrounds could also potentially be used in a GWAS. An advantage of using such a mixture population is that it should improve the detection of causal variants by increasing the sample size. Conversely, a GWAS with a mixture population may suffer from large numbers of false positives caused by the population structure. Although a mixed effect model that suppresses the influence of the population structure has been proposed (Yu et al., 2006), such a mixture population has rarely been analyzed by a GWAS.

An actual data analysis of rice using whole-genome sequences showed that the detection power of a GWAS improved when Oryza sativa subsp. japonica and Oryza sativa subsp. indica populations were combined (Misra et al., 2017). Furthermore, the identification of new rice genes using a GWAS and populations with extremely high genetic diversities has also been previously reported (Zhao et al., 2011). Conversely, it has been reported that the genetic differentiation between subpopulations in a population with high genetic diversity could cause a reduction in the power of a GWAS (Huang et al., 2012). Therefore, real data studies have been 
104 inconsistent about whether mixture populations or populations with high genetic diversities

105 should be used in a GWAS. However, these previous studies mostly analyzed actual data, and

106 there have been no theoretical simulation studies that have considered the possibility of using a

107 mixture population in a GWAS. Furthermore, no previous studies have discussed which kinds of

108 populations should be mixed to improve the GWAS detection power or which kinds of

109 populations are most appropriate for a GWAS. Therefore, in this study, we conducted simulation

110 experiments to see whether adding a population with a high genetic diversity compared to a

111 target population (e.g., adding a diversity panel to a target population) is appropriate, especially

112 when the genetic diversity of the target population is small.

\section{MATERIALS AND METHODS}

\section{Materials (populations used in the GWAS)}

In this study, 112 accessions of Oryza sativa subsp. japonica (referred to as "A"), which

117 were accessions that had mainly been developed in Japan, were used as a target population with

118 low genetic diversity (Yabe et al., 2016). We used the following three populations selected from

119 "The 3,000 Rice Genomes Project" (J. Y. Li et al., 2014), i.e., 100 accessions of Oryza sativa

120 subsp. indica (referred to as "B"), 100 accessions of Oryza sativa subsp. japonica (referred to as

121 "C" or temperate), and 100 accessions of Oryza sativa with various genetic backgrounds

122 (referred to as "D" or diverse), as populations with higher diversities than the target population

123 (Table S1 in Supplemental File 1). The process used to select each of the 100 accessions is

124 described in Supplemental File 9. One accession (IRIS ID: IRIS 313-11868) was duplicated in

125 populations B and D. Among populations B, C, and D, the B population was the most 
126 differentiated from A, whereas $\mathrm{C}$ was the most similar to A. Population D contained subsp.

127 indica, subsp. japonica, and aus, and aromatic rice accessions, which meant that the D

128 population had the highest genetic diversity. Fig. 1 is an unrooted phylogenetic tree that shows

129 the genetic relationships among accessions belonging to populations A, B, C, and D. 


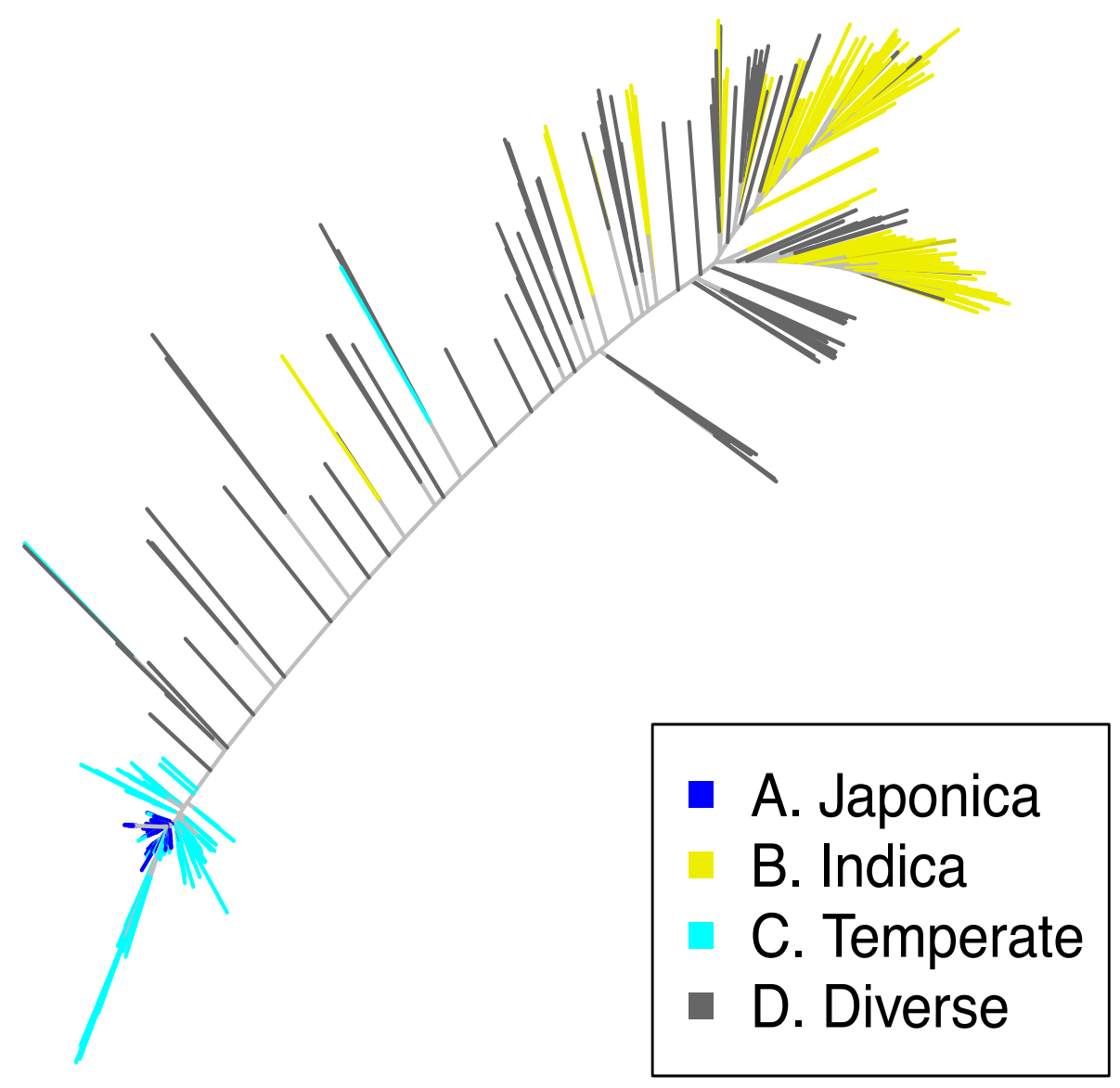

132 Fig. 1. Unrooted phylogenetic tree plot for four non-mixture populations.

133 Unrooted phylogenetic tree plot for the four non-mixture populations, which consisted of 112

134 accessions of japonica (A), 100 accessions of indica (B), 100 accessions of temperate japonica

135 (C), and 100 diverse accessions (D) with neighbor-joining method. 
The genetic relationships among the accessions were estimated by the neighbor-joining (NJ)

genetic distances were estimated according to the Jukes and Cantor (1969) model. In addition to

populations B, C, or D. The mixture populations A + B, A + C, and A + D were named "E", "F", and "G", respectively. We compared the QTN detection power the GWAS when the seven nonmixture (A, B, C, and D) and mixture populations (E, F, and G) were used.

\section{Genotype data}

Whole genome sequencing data were available for the accessions (Jarquin et al., 2019).

Details about the DNA extraction and whole genome sequencing techniques are provided in a previous report (Jarquin et al., 2019). The data sets deposited in the DDBJ Sequence Read Archive (SRA106223, ERA358140, DRA000158, DRA000307, DRA000897, DRA000927, 
159

160

161

162

163

164

165

166

167

168

169

170

171

\begin{tabular}{lllll}
\hline & Population name & Number of accessions & Number of SNPs & Diversity level $\dagger$ \\
\hline A. & Japonica & 112 & 72,110 & 263.095 \\
B. & Indica & 100 & 427,943 & 660.416 \\
C. & Temperate japonica & 100 & 135,665 & 362.649 \\
D. & Diverse & 100 & 647,731 & 798.646 \\
E. & A + B & 212 & 633,507 & 803.064 \\
F. & A + C & 212 & 151,675 & 334.606 \\
G. & A + D & 212 & 684,774 & 859.678
\end{tabular}

al., 2014) and Picard package V2.5.0 (http://broadinstitute.github.io/picard). The mapped reads were realigned using RealignerTargetCreator and indelRealigner in the GATK software. The SNPs and InDels were called at the population level using the UnifiedGenotyper in GATK and the -glm BOTH option. We extracted bi-allelic sites in all the accessions from the variants using VCFtools version 0.1.13 (Danecek et al., 2011). Then, imputations were imputed using Beagle version 4.1 (Browning and Browning, 2016). Finally, we analyzed the SNPs with minor allele frequencies $(\mathrm{MAFs}) \geq 0.05$ in each population. In the analysis, the genotypes were represented as -1 (homozygous of the reference allele), 1 (homozygous of the alternative allele) or 0 (heterozygous of the reference and alternative alleles). Out of all the whole-genome sequence polymorphisms, only the SNPs on chromosome 1 were analyzed. The number of SNPs on chromosome 1 in each population is shown in Table 1.

Table 1. Number of SNPs and the diversity level of non-mixture and mixture populations. $\dagger$ Diversity level is the index that was used to indicate the degree of genetic diversity and is

173 described in the "Degree of genetic diversity index" section below. 


\section{Generating phenotype data}

177
Phenotypic data were simulated using the following formula:

$$
\mathbf{y}=\mathbf{X}_{1} \beta_{1}+\mathbf{X}_{2} \beta_{2}+\mathbf{X}_{3} \beta_{3}+\mathbf{u}+\mathbf{e}
$$

where $\mathbf{y}$ is the vector that represents the simulated phenotypic values for all 411 accessions; $\mathbf{X}$ is the design matrix representing the genotypes of three quantitative trait nucleotides (QTNs) with scores $-1,0$, or $1 ; \boldsymbol{\beta}=\left[\begin{array}{lll}\beta_{1} & \beta_{2} & \beta_{3}\end{array}\right]^{\mathrm{T}}$ is the vector representing the effects of the three QTNs, $\mathbf{u}$ is the vector for polygenetic effects, and $\mathbf{e}$ is the residuals vector. Three QTN-SNPs whose MAF was equal to or larger than 0.05 in all 411 accessions (672,923 SNPs in total) were randomly selected from the SNPs on chromosome 1. The simulations were divided into five categories (low, lower-middle, middle, higher-middle, high) based on the fixation index $\left(F_{S T}\right)$ between populations A and B for the first QTN (Fig. S1 in Supplemental File 2). We assumed that the first QTN had four times greater variance than the remaining two QTNs (referred to as "QTN1", “QTN2”, and "QTN3” respectively). The remaining two QTNs were chosen randomly from SNPs where the $F_{S T}$ between A and B were low (SNPs whose $F_{S T}$ value was in the lower 20\% category among the $672,923 \mathrm{SNPs})$. The $F_{S T}$ for each marker was calculated according to Wright (1965) as follows:

$$
F_{S T}=1-\frac{H_{S}}{H_{T}}
$$


193 where $H_{S}$ is the average of the expected heterozygosity based on the allele frequencies of

194 populations $\mathrm{A}$ and $\mathrm{B}$, and $H_{T}$ is the expected heterozygosity based on the average allele

195 frequency of populations $\mathrm{A}$ and $\mathrm{B} . H_{S}$ and $H_{T}$ were calculated as follows:

$$
H_{S}=\frac{N_{A} \cdot\left\{2 p_{A}\left(1-p_{A}\right)\right\}+N_{B} \cdot\left\{2 p_{B}\left(1-p_{B}\right)\right\}}{N_{A}+N_{B}},
$$

$$
H_{T}=2\left(\frac{N_{A} p_{A}+N_{B} p_{B}}{N_{A}+N_{B}}\right)\left(1-\frac{N_{A} p_{A}+N_{B} p_{B}}{N_{A}+N_{B}}\right),
$$

where $p_{A}, p_{B}, N_{A}$, and $N_{B}$ are the allele frequencies and the sample sizes of populations A and B respectively, and $N_{A}=112$ and $N_{B}=100$. The $F_{S T}$ distribution between $\mathrm{A}$ and B is shown in Fig. S1, which also shows the thresholds for the five $F_{S T}$ categories.

The polygenetic effect in Eq. 5 was sampled from the multivariate normal distribution whose variance-covariance matrix was proportional to the additive numerator relationship matrix A and was normalized so that their variance was equal to that of the three QTN effects.

$$
\mathbf{u} \sim \operatorname{MVN}(0, \mathbf{G}),
$$

where $\mathbf{G}=\mathbf{A} \sigma_{\mathrm{A}}^{2}$ is the genetic covariance matrix, and the additive genetic variance $\sigma_{\mathrm{A}}^{2}$ was automatically determined from the relationship with heritability. In this study, the additive numerator relationship matrix A was estimated based on the marker genotype data for 402,509 SNPs, which consisted of the core SNPs (defined by the Rice SNP-Seek Database as the " $404 \mathrm{k}$ CoreSNP Dataset") in all 12 chromosomes (this marker genotype data was prepared separately from the whole-genome sequence data), using the "A.mat" function in R package "rrBLUP" version 4.5 (Endelman and Jannink, 2012; Endelman, 2011). 
212 The residual e in Eq. 6 was sampled identically and independently from the normal distribution,

213 and was then normalized so that the narrow-sense heritability was equal to 0.6 . Residual e was

214 calculated using the following formula:

$$
\mathbf{e} \sim \operatorname{MVN}\left(0, \mathbf{I} \sigma_{\mathrm{e}}^{2}\right)
$$
linear mixed model (Yu et al., 2006).

$$
\mathbf{y}=\mathbf{X} \boldsymbol{\beta}+\mathbf{S}_{i} \alpha_{i}+\mathbf{Q v}+\mathbf{Z u}+\mathbf{e}
$$

where $\mathbf{y}$ is the vector of phenotypic values, $\mathbf{X} \boldsymbol{\beta}, \mathbf{S}_{i} \alpha_{i}$, and $\mathbf{Q v}$ are the fixed effects terms, $\mathbf{Z u}$ is the random effects term, and $\mathbf{e}$ is the residuals vector. $\boldsymbol{\beta}$ represents all of the fixed effects other than $\mathbf{S}_{i} \boldsymbol{\alpha}_{i}$, and $\mathbf{Q} \boldsymbol{v}$, and $\mathrm{X}$ is the incidence design matrix corresponding to $\boldsymbol{\beta}$. In this study, $\mathbf{X} \boldsymbol{\beta}$

228 was an intercept. $\mathbf{S}_{i} \alpha_{i}$ is composed of $\mathbf{S}_{i}$, which is the $i_{\text {th }}$ marker of the genotype data, and $\alpha_{i}$,

229 which is the effect of that marker. Qv is the term used to correct for the effect of population

230 structure, and in this study $\mathbf{Q}$ was the matrix of two eigenvectors corresponding to the upper two

231 eigenvalues of the additive numerator for relationship matrix $\mathbf{A}$, Finally, $\mathbf{u}$ represents the

232 polygenetic effects, and $\mathbf{Z}$ is the incidence design matrix corresponding to $\mathbf{u}$. 
We used the EMMAX and P3D algorithms to reduce the computation time (Kennedy et al., 1992; Kang et al., 2008; 2010; Zhang et al., 2010). The “GWAS” function in R package “rrBLUP” version 4.5 (Endelman, 2011) was used to perform the GWAS described above.

\section{Evaluation of the simulation results}

The $p$-value (or $-\log _{10}(p)$ ) for each marker effect was estimated 100 times by the GWAS in five patterns according to the size of the $F_{S T}$ for the seven non-mixture/mixture populations. In this study, the following summary statistics were mainly used to evaluate the GWAS results.

In the 100 simulations, the QTNs were not always polymorphic in each population (because the MAF of the whole population did not necessarily match the MAF of each individual population). In such cases, the $-\log _{10}(p)$ value of a QTN that was not polymorphic within a population could not be calculated. Therefore, when two SNPs were polymorphic within that population and were adjacent to the QTN, then the statistic of the more significant SNP was used as the QTN statistic. Since it was difficult to detect such QTNs using a GWAS, we calculated the summary statistics by dividing two patterns depending on polymorphism patterns of QTN1, i.e., whether using all simulation results or using only results whose QTN1 was polymorphic in the target population (referred to as "All" and "Polymorphic in the population", respectively). 


\section{Correct detection rate (CDR) and $-\log _{10}(p)$}

The first summary statistic was whether the $-\log _{10}(p)$ rate for each QTN exceeded the threshold in each GWAS (referred to as "CDR; correct detection rate"). We assumed that QTNs would be successfully detected by the GWAS when the CDR was large. The $-\log _{10}(p)$ value whose false discovery rate (FDR) was 0.05 was set as the threshold using the Benjamini-

Hochberg method (Benjamini and Hochberg, 1995; Storey and Tibshirani, 2003). As the second summary statistic, we used the $-\log _{10}(p)$ for each QTN in each GWAS, and we also assumed that QTNs were successfully detected by the GWAS when this statistic was large.

\section{Area under the curve (AUC)}

We also regarded the mean of the AUC as one summary statistic. The AUC refers to the area under the receiver operating characteristic (ROC) curve (Fig. S2 in Supplemental File 3), which was obtained by plotting the false positive rate on the horizontal axis and the true positive rate on the vertical axis when the threshold was varied (Hanley and McNeil, 1982). The AUC was calculated using the following formula:

$$
\mathrm{AUC}=\frac{1}{2} F P R s_{1} T P R s_{1}+\frac{1}{2} \sum_{i=2}^{m+1}\left(F P R s_{i}-F P R s_{i-1}\right)\left(T P R s_{i}+T P R s_{i-1}\right)
$$

where $m$ is the number of QTNs, and $m=3$ in this study. The FPRs and TPRs are the $m+1$ vectors whose $i_{\text {th }}$ elements are $F P R s_{i}$ and $T P R s_{i}$, respectively. $F P R s_{i}=T P R s_{i}=1$ when $i=$ $m+1$. When $1 \leqq i \leqq m$, the $F P R s_{i}$ and $T P R s_{i}$ represent the false positive rate and the true positive rate at the time when $i$ QTNs exceed the threshold, respectively. They were calculated using the following formula: 


$$
F P R s_{i}=\frac{F P_{i}}{F P_{i}+T N_{i}} \quad(1 \leqq i \leqq m),
$$

$$
T P R s_{i}=\frac{T P_{i}}{T P_{i}+F N_{i}} \quad(1 \leqq i \leqq m),
$$

276 where $T P_{i}, F P_{i}, F N_{i}$, and $T N_{i}$ are the numbers of SNPs that are the true positives (where the SNP

277 is a QTN and exceeds the threshold), the false positives (where the SNP is not a QTN but

278 exceeds the threshold), the false negatives (where the SNP is a QTN but does not exceed the

279 threshold), and the true negatives (where the SNP is not a QTN and does not exceed the

280 threshold) at the time when $i$ QTNs exceed the threshold respectively. When we evaluated the

281 true/false positive rate, we considered the existence of linkage disequilibrium (LD) by

282 investigating SNPs with LD as one set. In this study, we defined SNPs that satisfied the

283 conditions that they were within $300 \mathrm{~kb}$ from the focused SNP and the condition that their

284 squares of the correlation coefficients with the focused SNP were 0.35 or more as one set when

285 considering LD. When we counted $T P_{i}, F P_{i}, F N_{i}$, and $T N_{i}$, we counted the number of the sets

286 described above instead of the number of SNPs. The value for AUC calculated in this manner

287 takes a value between 0 and 1. The GWAS is more successful when the AUC is closer to 1.

288 Using the mean of the AUC as one of the summary statistics meant that it was possible to focus 289 on each QTN and evaluate the overall results of the GWAS.

\section{Precision, recall, and F-measure}


295 calculated from the numbers of true positives, false positives, false negatives, and true negatives.

296 More specifically, the precision can be calculated using the following formula:

$$
\text { Precision }=\frac{T P}{T P+F P} \text {. }
$$

QTNs. The recall was defined using the following formula:

$$
\text { Recall }=\frac{T P}{T P+F N}
$$
formula:

$$
F=\frac{2 \cdot \text { Precision } \cdot \text { Recall }}{\text { Precision }+ \text { Recall }}
$$

\section{Degree of genetic diversity index}

In order to evaluate the relationship between genetic diversity and the CDR results, we 
314 Euclidean distance matrix between accessions for each population was calculated. The median

315 for the off-diagonal elements of the distance matrix was used to indicate the degree of genetic

316 diversity (referred to as the "diversity level", Table 1). The median was chosen as the diversity

317 level because the distribution of the distances between the accessions for $\mathrm{E}$ and $\mathrm{G}$ had a double

318 peak. This was because, for mixture populations such as $\mathrm{E}$ and $\mathrm{G}$, the distance within the

319 subpopulations was small whereas the distance between subpopulations was large. Therefore, if

320 the mean of the distances (almost the same as Nei's gene diversity index (NEI, 1973)) is chosen

321 as the diversity level, then there is a risk of overestimating the diversity level.

322

\section{RESULTS}

\section{Comparisons between the CDR and AUC for the QTN1s in each population}

The CDRs of the QTN1s in each population were calculated under ten conditions: five levels

327 of $F_{S T}$ between A and B and two patterns of QTN polymorphism, i.e., whether the QTN was

328 polymorphic or not in the target population (Fig. 2 and Table S2 in Supplemental File 5). 

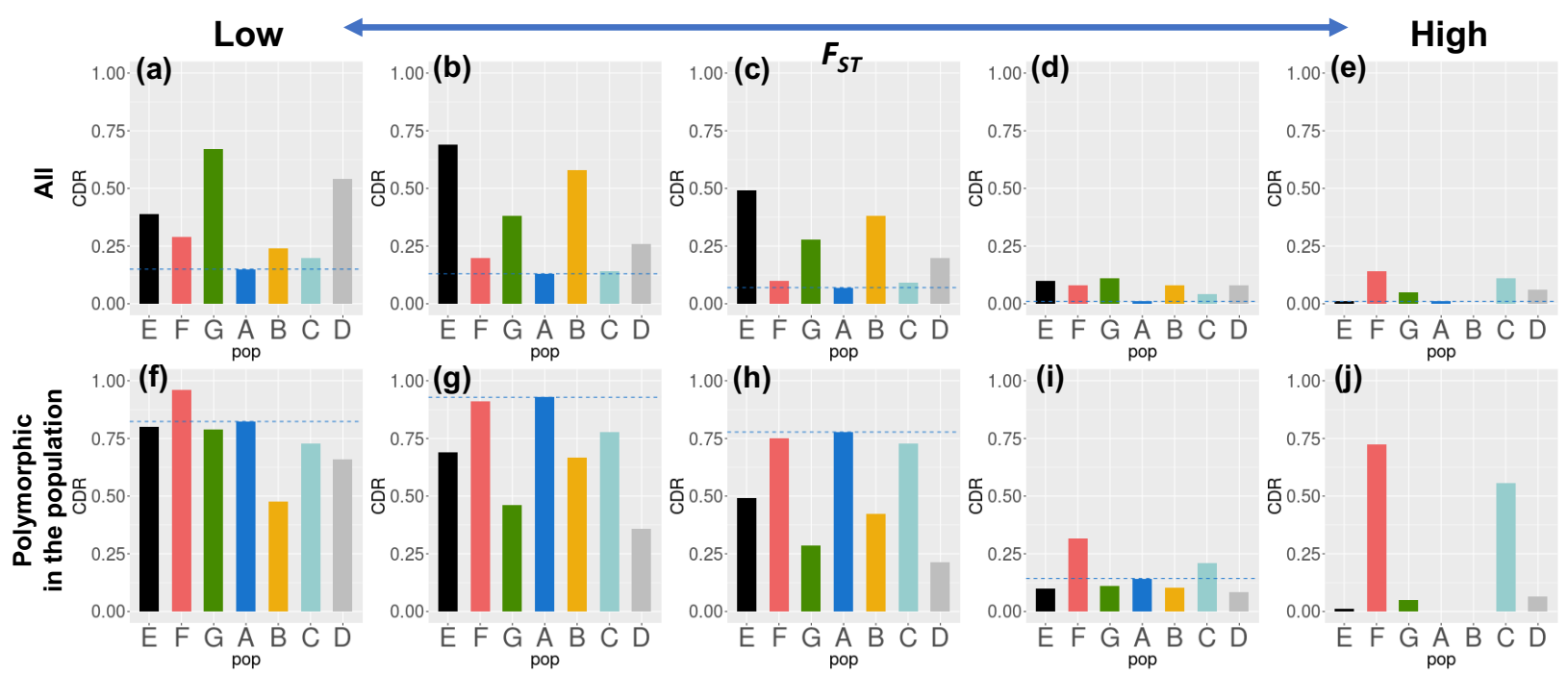

Fig. 2. Correct detection rate for QTN1 in each population under ten conditions.

333 The barplots of CDR of QTN1 in each population under ten conditions: five levels of $F_{S T}$ of QTN1 and

334 two patterns of polymorphisms of QTN. Blue horizontal dashed lines indicate the CDR in the population

335 A for each population. A: japonica, B: indica, C: temperate japonica, D: diverse, E: A+ B, F: A + C, G:

$336 \mathrm{~A}+\mathrm{D}$. 
For almost all levels of $F_{S T}$, the CDRs for QTN1 in the mixture populations E, F, and G

$\mathrm{G}$ were always larger than in population A when all the simulation results were taken into

QTN1 was hardly detected because of the strong confounding effect of the population structure.

In the other populations, the expected heterozygosity $\left(H_{e}\right)$ for QTN1 was extremely small (In A

We excluded the simulations in which there were no polymorphisms in the population so that the detection power of the GWAS when there were polymorphisms in an analyzed population could be evaluated (Figs. $2 \mathrm{f}-\mathrm{j}$ ). When $F_{S T}$ was low, population $\mathrm{F}$ had the highest CDR

358 and when $F_{S T}$ was in the lower-middle or middle categories, population A had the highest CDR.

359 However, there were only 14 and 9 cases in which QTN1 was polymorphic in population A. In 
361 than the populations with high genetic diversities (D, E, and G). When $F_{S T}$ was in the higher-

362 middle or high categories, the results were similar to when $F_{S T}$ was in the lower-middle or

363 middle categories.

The CDRs of QTN2 and QTN3 were much lower than that of QTN1 because smaller genetic variances were assigned to these QTLs than QTN1 (Table S2). As in the case of QTN1, higher. smaller AUC than populations D and G, even when the CDR was highest in population F. when the $F_{S T}$ of QTN1 was high. 
(a) E. Japonica + Indica

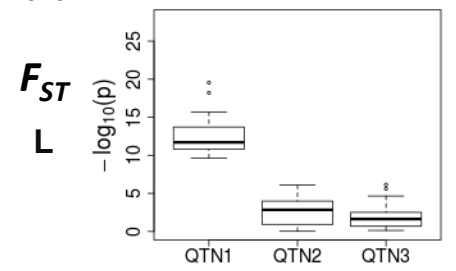

(b)

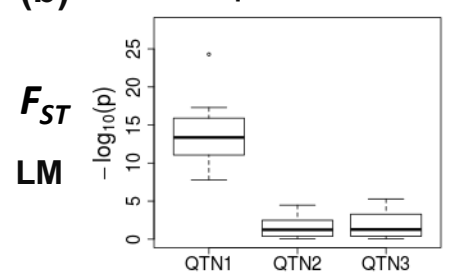

(c)

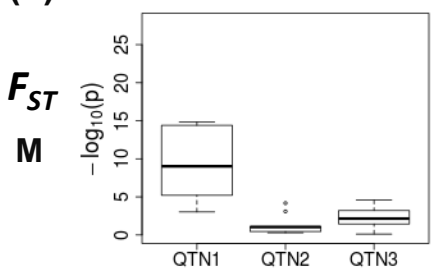

(d)

E. Japonica + Indica

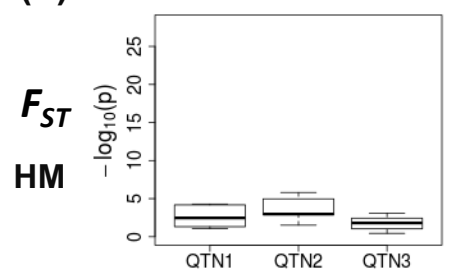

F. Japonica + Temperate

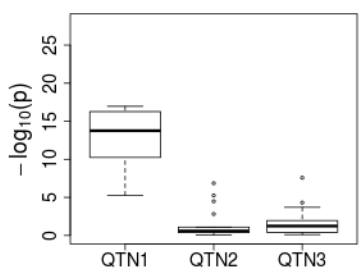

F. Japonica + Temperate

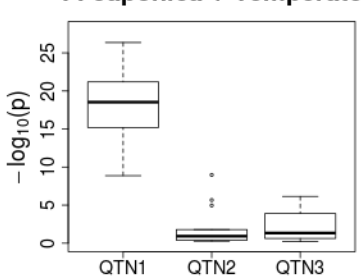

F. Japonica + Temperate



F. Japonica + Temperate

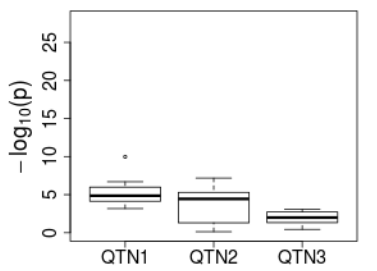

G. Japonica + Diverse

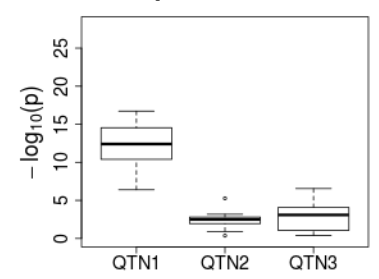

G. Japonica + Diverse

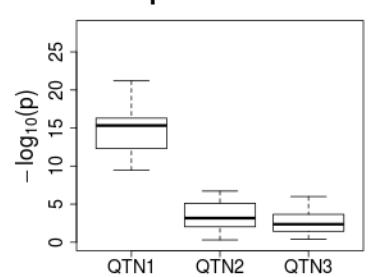

G. Japonica + Diverse

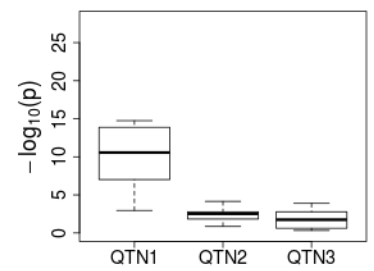

G. Japonica + Diverse

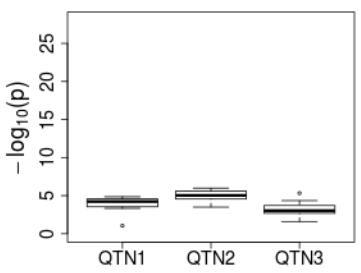

A. Japonica

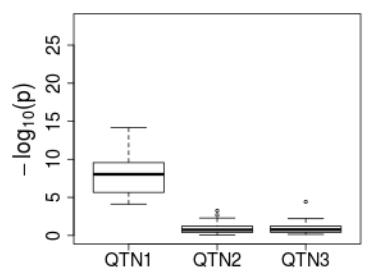

A. Japonica

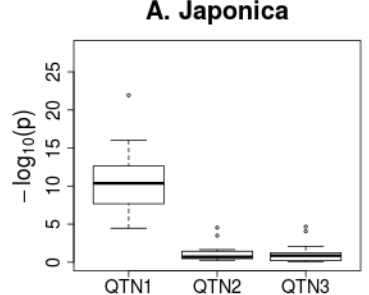

A. Japonica

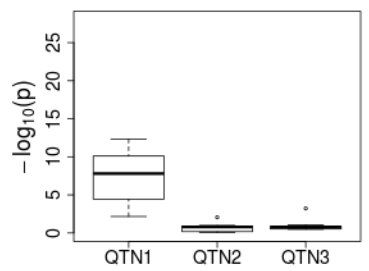

A. Japonica

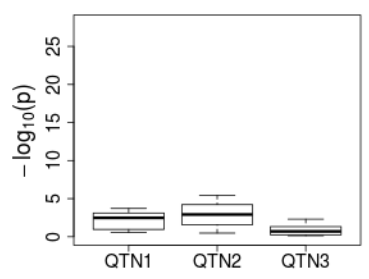

Fig. 3. Boxplots of $-\log _{10}(p)$ of each QTN when QTN1 was polymorphic in japonica (A). 
391 conspicuous even when $F_{S T}$ was in the middle or higher-middle categories (Figs. 3c, d,

392 respectively). This is because the QTN1 effect is less likely to be confounded with the population

393 structure in $F$ than in the other mixture populations ( $E$ and $G)$. Population $G$ had the highest

$394-\log _{10}(p)$ values for QTN2 and QTN3, although only slightly (Fig. 3).

\section{Factors affecting the detection power of QTNs in the mixture populations}

398 We considered the factors related to the detection power of QTNs in the mixture populations

399 by creating a figure that represented the relationship between $F_{S T}$, the expected heterozygosity

$400\left(H_{e}\right)$, and the QTN1 detection power (Fig. 4 and Fig. S4 in Supplemental File 6). 
(a) E. Japonica + Indica

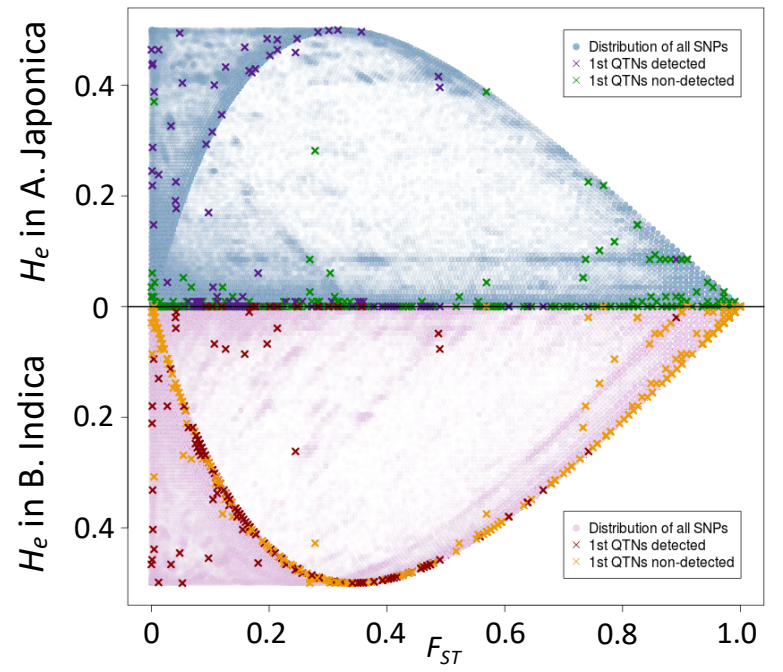

(b) F. Japonica + Temperate

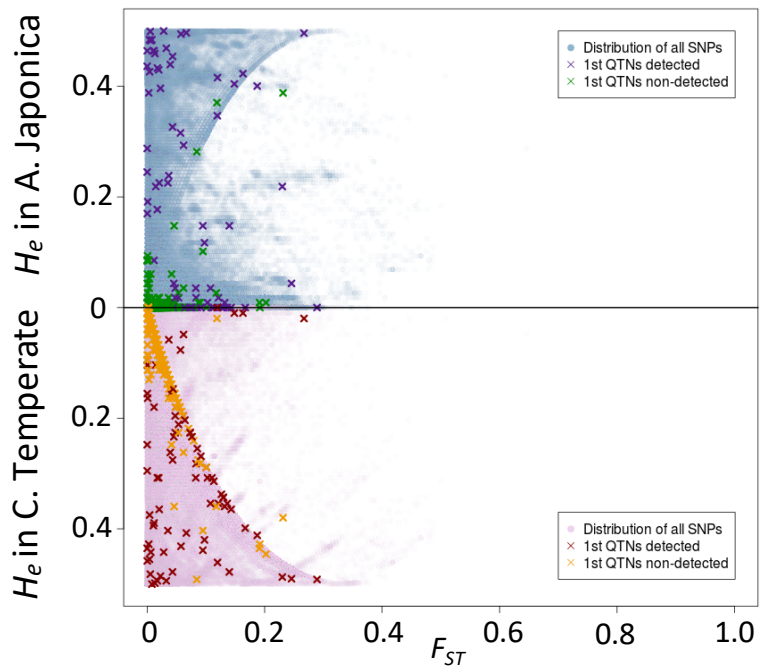

403

Fig. 4. Relationship between $\boldsymbol{F}_{\boldsymbol{S T}}, \boldsymbol{H}_{e}$, and the detection power of QTN1.

404 The distribution of each marker is plotted thinly with between subpopulation $F_{S T}$ on the

405 horizontal axis and $H_{e}$ of each subpopulation on the vertical axis. The dark X marks on the plot

406 show the SNPs selected as QTN1s in this study. Red and purple marks were detected by GWAS,

407 and green and yellow ones were not detected by GWAS. 
411 difference between plots F and E or G (Fig. 4a, Fig. S4, and Fig. 4b). However, in population F,

412 because $\mathrm{A}$ and $\mathrm{C}$ are genetically close, the $F_{S T}$ between the subpopulations was not high.

413 Therefore, the relationships between $F_{S T}, H_{e}$, and the GWAS detection power applied to all

414 mixture populations.

Some of the QTNs were detected by the GWAS when $F_{S T}$ was in the medium category, and subpopulation was added to the analysis.

\section{Comparisons among the precision, recall, and $\boldsymbol{F}$-measure values for each population}


431 Finally, a comparison of the $F$-measure for each population showed that there seemed to be no

432 tendency associated with $F_{S T}$. Therefore, it was difficult to conclude which population was

433 suitable for a GWAS when the $F$-measure is used. These results indicated that using mixture

434 populations for a GWAS led to the detection of more SNPs, including QTNs.

435

\section{Relationship between the CDR results and genetic diversity}

437

438

439

440

441

442

443

444

445

446

447

448

449

450

451

The relationship between the CDR results for QTN1 and the degree of genetic diversity was evaluated under the two QTN polymorphism patterns, i.e., whether or not QTN was polymorphic in the population (Fig. S6 in Supplemental File 8). The CDRs for the mixture populations were usually larger than for the non-mixture populations if their diversity levels were close (Fig. S6a, b). A comparison of the results for the different $F_{S T}$ categories showed that when $F_{S T}$ was low, the populations with the highest diversities, such as D or G, had the highest CDRs, and when $F_{S T}$ was in the lower-middle or middle categories, the populations with the second-highest diversities, such as B or E, had the highest CDRs. Finally, when $F_{S T}$ was in the higher-middle or high categories, the populations with relatively low diversities, such as $\mathrm{C}$ or $\mathrm{F}$, had the highest CDRs (Fig. S6a). However, when the simulations in which there were no polymorphisms in the population were excluded, the populations with relatively low diversities, such as A, C, or F, had the highest CDRs in almost all the $F_{S T}$ categories (Fig. S6b). 


\section{DISCUSSION}

\section{Relationship between $F_{S T}$ and QTN detection}

454 One of the main results of this study was that the detection of QTNs was difficult in populations

455 with high genetic diversities, such as D, E, and G, when the $F_{S T}$ for QTN1 between japonica (A)

456 and indica (B) was high. This was because the QTN effect confounds with the effect of

457 population structure in these populations. We also examined the reasons why the CDRs for

458 QTN2 and QTN3 were high when the QTN1 $F_{S T}$ value was high.

In this study, phenotypic values were simulated using the following expression:

$$
\mathbf{y}=\mathbf{X}_{1} \beta_{1}+\mathbf{X}_{2} \beta_{2}+\mathbf{X}_{3} \beta_{3}+\mathbf{u}+\mathbf{e}
$$

where $\mathbf{u}$ is the polygenetic effect, and is the term that reflects differences between accessions and thus differences between subpopulations. Therefore, if the degree of QTN1 genetic differentiation between japonica (A) and indica (B) is high, it can be assumed that there is a high correlation between $\mathbf{X}_{1} \beta_{1}$ and $\mathbf{u}$. In this study, we generated phenotypic values using a certain variance ratio under the assumption that each term is independent. Therefore, if there is a correlation between $\mathbf{X}_{1} \beta_{1}$ and $\mathbf{u}$, and the variance between these two terms is considered as one unit, it can be assumed that the variance is smaller than the total value of the two variances under the assumption of independence. Therefore, the variance of these two terms $\left(\mathbf{X}_{1} \beta_{1}+\mathbf{u}\right)$ in the total phenotypic variance becomes smaller, whereas the variances caused by the terms $\mathbf{X}_{2} \beta_{2}$ and $\mathbf{X}_{3} \beta_{3}$ become greater than those when it is assumed that each term is independent.

The GWAS model used in this study was

$$
\mathbf{y}=\mathbf{X} \boldsymbol{\beta}+\mathbf{S}_{i} \alpha_{i}+\mathbf{Q} \mathbf{v}+\mathbf{Z} \mathbf{u}+\mathbf{e},
$$


473 where Qv is the term used to correct the effect of population structure, and Zu shows the

474 polygenetic effect. In this GWAS model, $\mathbf{S}_{i} \alpha_{i}$ and $\mathbf{Q v}$ or $\mathbf{Z u}$ have some correlation when $\mathbf{S}_{i}=$

$475 \mathbf{X}_{1}$. This correlation results in the underestimation of $\alpha_{i}$ by the terms originally used to correct

476 the effects of population structure or family relatedness, such as Qv and Zu. Therefore, QTN

477 detection is quite difficult when a GWAS is performed on mixture populations. For QTN2 and

478 QTN3, where $\mathbf{S}_{i}=\mathbf{X}_{2}$ or $\mathbf{S}_{i}=\mathbf{X}_{3}$, there is generally no correlation between $\mathbf{S}_{i} \alpha_{i}$ and $\mathbf{Q v}$ or $\mathbf{Z u}$.

479 Therefore, the detection of these QTNs is not related to these terms. Furthermore, the variances

480 represented by the terms $\mathbf{X}_{2} \beta_{2}$ and $\mathbf{X}_{3} \beta_{3}$ are considered to be higher when the QTN1 genetic

481 differentiation is not high. Therefore, the CDRs of QTN2 and QTN3 were high when the $F_{S T}$ for

482 QTN1 was high (Fig. 2 and Table S1). It has been suggested by Atwell et al. (2010) that a bias

483 may occur in the GWAS results when the QTN correlates with population structure or family

484 relatedness.

485

486

\section{Relationship between $H_{e}$ and the QTN detection}

488 The detection of QTNs by a GWAS was difficult when the expected heterozygosity $\left(H_{e}\right)$ in the

489 population was low. When $H_{e}$ in the population was low, the MAF was low, and alleles and

490 mutations with low allele frequencies are known as "rare alleles" or "rare variants". In such cases,

491 the QTN effect when the $H_{e}$ values are low may be concealed by the QTN effect when $H_{e}$ is not

492 low or by the environmental effect because there are few accessions with one allele. For this

493 reason, it is generally challenging to detect QTNs in such cases, but a method to deal with this

494 problem has been developed (Wu et al., 2011). 
One example of a "rare variant" that is common in plants is the haplotype condition. It has

496

497

498

499

500

501

502

503

504

505

506

507

508

509

510

511

512

513

514

515

516

517

been reported that haplotypes are difficult to detect using a GWAS (Stram, 2014). This is

because haplotypes are often "rare variants" and their $H_{e}$ values in the population are low.

Another problem caused by "rare variants" is that the non-causal SNP whose LD is strong with the "rare variant" may have a higher $-\log _{10}(p)$ value than the "rare variant". This occurrence, known as "synthetic association", often happens when the minor allele frequency of the SNP is higher than that of the "rare variant" (Dickson et al., 2010). These "synthetic associations" were often detected in this simulation study.

\section{Summary and further discussion on each result}

Generally, the CDRs of the QTNs showed that the populations suitable for a GWAS were different depending on whether all the QTNs were to be detected or only the polymorphic QTNs in the target population. Specifically, if all QTNs are to be detected when the degree of genetic differentiation between QTNs is low, then it is optimal to use a population with high genetic diversity that has as many polymorphisms as possible. However, as the degree of genetic differentiation becomes more extensive, a population with high genetic diversity is not suitable for a GWAS because the QTN effect is more likely to confound with the population structure. In contrast, a population with moderate genetic diversity, such as population $\mathrm{F}$, was suitable for a GWAS, regardless of the degree of genetic differentiation. This was partly because the QTN1 effect was less likely to confound with the population structure in $\mathrm{F}$ than in $\mathrm{E}$ or $\mathrm{G}$, even when $F_{S T}$ was high. However, in either case, when the degree of genetic differentiation is extensive, it is difficult to detect the QTNs in any population. Therefore a GWAS analysis is not suitable, 
518 which means that another approach, such as biparental QTL mapping, must be used to identify

519 genes (Lander and Botstein, 1989).

521 population $\mathrm{F}$ was the highest. From its definition, AUC is more dependent on how low

$522-\log _{10}(p)$ of the QTN with the lowest $-\log _{10}(p)$ value is than on how high the $-\log _{10}(p)$ of

523 the QTN with the highest $-\log _{10}(p)$ value is. Furthermore, in this study, the number of markers

524 for the GWAS differed (Table 1). When $-\log _{10}(p)$ values for the QTNs were similar among the

525 different populations, the larger number of markers meant that the true negative rate increased,

526 and the false positive rate decreased in a population, which resulted in an increase in the AUC of

527 a population with a larger number of markers, e.g. D and G.

A comparison of the mixture populations and japonica (A) using $-\log _{10}(p)$ showed

529 that when the QTNs are polymorphic in a target population with low genetic diversity, genetic

530 resources with higher genetic diversities should be added to the target population. However, in

531 order to avoid cases where the degree of genetic differentiation among the QTNs is extensive

532 between the target population and genetic resources, it is desirable to use populations that are

533 genetically close to the target population.

Finally, the results suggested that the $F_{S T}$ differences between the subpopulations and the expected heterozygosity $\left(H_{e}\right)$ of each subpopulation greatly influenced QTN detection by the

536 GWAS in the mixture populations (Fig. 4 and Fig. S4). This result was in agreement with the

537 above finding that QTN detection using a GWAS was generally difficult when $F_{S T}$ was high, or

$538 H_{e}$ were low. However, these situations frequently happened when the $F_{S T}$ between the

539 subpopulations was moderate. Therefore, even if a QTN is fixed in one subpopulation, it may be 
540 possible to detect the QTN by adding another population to the analysis because when the $H_{e}$ of

541 the QTN is low in one population and $F_{S T}$ is moderate, it can be assumed that $H_{e}$ is relatively

542 high in the other population. Therefore, the $H_{e}$ of the mixture population as a whole becomes

543 larger and the detection of a QTN is possible unless the confounding of the effect of that QTN

544 with the population structure is extensive. Although this situation is not difficult to interpret, it is

545 extremely important that SNPs with high $F_{S T}$ and low $H_{e}$ values must exist in large numbers

546 among populations. After taking this fact into account, a GWAS with a mixture population can

547 be useful. Therefore, creating the proposed diagram shown in Fig. 4 and Fig. S4, will lead to a

548 quantitative understanding of what kind of SNPs can be detected by a GWAS in mixture

549 populations of interest.

\section{Relationships with using whole-genome sequences}

555 (B) subpopulations was low, the CDR of the mixture populations was high. One example of such

556 markers is that mutations may have occurred at the same position in both populations after they

557 differentiated. Since such variants are relatively new variants, the LD relationship between these

558 variants and surrounding markers will be weak. Therefore, these variants cannot be detected

559 using marker genotype data with a small number of markers, such as an SNP array. However, the

560 use of whole-genome sequences will increase the marker density, which improves the possibility

561 of detecting such variants with a GWAS. In summary, using whole-genome sequences improves 
562 the possibility of detecting QTNs with low $F_{S T}$ values and the use of mixture populations should

563 further improve the QTN detection power. In this study, there were cases where SNPs in a low

564 LD region were selected as QTNs when $F_{S T}$ was low.

565

566

567

568

569

570

571

572

573

574

575

576

577

578

579

580

581

582

\section{CONCLUSION}

In this study, we examined a way of selecting a population that was suitable for a GWAS by conducting simulations using populations with various genetic backgrounds. We evaluated the results of the simulations by dividing them into ten patterns according to two criteria: the degree of genetic differentiation $\left(F_{S T}\right)$ between two main subpopulations and QTN polymorphism in a target population. When the QTNs are polymorphic in a target population, increasing the population size by adding available genotypes to the target population improves the detection power. We suggest that a population genetically similar to a target population is desirable. After investigating $F_{S T}$ and expected heterozygosity $H_{e}$ as factors that may substantially influence the detection power of a GWAS, the results showed that SNPs with high $F_{S T}$ and low $H_{e}$ values were less likely to be detected by a GWAS that used mixture populations. These results indicated that the detection power of a GWAS was improved by using mixture populations with different genetic backgrounds. Furthermore, the use of publicly available whole-genome sequences meant it was possible to increase the population size and to use polymorphic markers that were present in high numbers, which should also improve the detection power of the GWAS. 


\section{ACKNOWLEDGMENTS}

590 in-Aid for Scientific Research(B) (Grant number 15H04436), JST, PRESTO (Grant number

591 JPMJPR15O6), the Cross-ministerial Strategic Innovation Promotion Program (SIP), the

592 "Technologies for creating next-generation agriculture, forestry and fisheries" (funding agency:

593 Bio-oriented Technology Research Advancement Institution, NARO), and JST CREST (Grant

594 Number JPMJCR16O).

597 Supplemental File 1: Table S1. Information about the 299 rice accessions used in this study.

598 Supplemental File 2: Fig. S1. Histogram showing the $F_{S T}$ differences between japonica (A) and indica 599 (B).

600 Supplemental File 3: Fig. S2. Example of a ROC curve and the AUC.

601 Supplemental File 4: Fig. S3. Principal components analysis results for chromosome 1 and all the 602 chromosomes.

603 Supplemental File 5: Table S2. Correct detection rate rates for all QTNs and the AUC in each population. 
604 Supplemental File 6: Fig. S4. Relationship between $F_{S T}, H_{e}$, and the QTN1 detection power for the

605 population $\mathrm{G}$.

606 Supplemental File 7: Fig. S5. Bar plots of the precision, the recall and the $F$-measure results.

607 Supplemental File 8: Fig. S6. Relationship between the diversity level and the CDR of QTN1.

608 Supplemental File 9: Supplementary Note. Additional information about the materials used in this study.

609

\section{OPTIONAL SECTIONS}

Availability of data and material

Whole genome sequencing data are available of 112 accessions of Oryza sativa subsp.

612 japonica in the DDBJ Sequence Read Archive (SRA106223, ERA358140, DRA000158,

613 DRA000307, DRA000897, DRA000927, DRA007273, DRA007256, and DRA008071). Whole

614 genome sequencing data for all the other accessions are available in the "Rice SNP-Seek

615 Database".

616

617 Competing interests

618 The authors declare that they have no competing interests.

619

620 Author's contributions

$621 \mathrm{KH}, \mathrm{HKK}$, and HI conceived and designed the study. KH and HI performed the

622 mathematical and statistical analysis. KH, HKK, MY, EK, SY and HN contributed to marker 
genotyping. KH, HKK, and $\mathrm{HI}$ wrote the manuscript in consultation with MY, EK, SY, and HN.

All authors read and approved the final manuscript.

Alexandrov, N., S. Tai, W. Wang, L. Mansueto, K. Palis, R.R. Fuentes, V.J. Ulat, et al. 2015. SNP-Seek Database of SNPs Derived from 3000 Rice Genomes. Nucleic Acids Res. 43(D1):D1023-27. doi: 10.1093/nar/gku1039.

Atwell, S., Y.S. Huang, B.J. Vilhjálmsson, G. Willems, M. Horton, Y. Li, D. Meng, et al. 2010. Genome-Wide Association Study of 107 Phenotypes in Arabidopsis Thaliana Inbred Lines. Nature 465(7298):627-31. doi: 10.1038/nature08800.

Auwera, G.A. Van Der, M.O. Carneiro, C. Hartl, R. Poplin, A. Levy-moonshine, T. Jordan, K. Shakir, et al. 2014. From FastQ Data to High Confidence Varant Calls: The Genonme Analysis Toolkit Best Practices Pipeline. Curr Protoc Bioinformatics 11(10):1-33. doi: 10.1002/0471250953.bi1110s43.From.

Begum, H., J.E. Spindel, A. Lalusin, T. Borromeo, G. Gregorio, J. Hernandez, P. Virk, B. Collard, and S.R. McCouch. 2015. Genome-Wide Association Mapping for Yield and Other Agronomic Traits in an Elite Breeding Population of Tropical Rice (Oryza Sativa). PLoS One 10(3):1-19. doi: 10.1371/journal.pone.0119873.

Benjamini, Y., and Y. Hochberg. 1995. Controlling the False Discovery Rate: A Practical and Powerful Approach to Multiple Testing. J. R. Stat. Soc. 57(1):289-300. doi: $10.2307 / 2346101$.

Bolger, A.M., M. Lohse, and B. Usadel. 2014. Trimmomatic: A Flexible Trimmer for Illumina Sequence Data 30(15):2114-20. doi: 10.1093/bioinformatics/btu170.

Browning, B.L., and S.R. Browning. 2016. Genotype Imputation with Millions of Reference Samples. Am. J. Hum. Genet. 98(1):116-26. doi: 10.1016/j.ajhg.2015.11.020.

Danecek, P., A. Auton, G. Abecasis, C.A. Albers, E. Banks, M.A. DePristo, R.E. Handsaker, et al. 2011. The Variant Call Format and VCFtools 27(15):2156-58. doi: 10.1093/bioinformatics/btr330.

Dickson, S.P., K. Wang, I. Krantz, H. Hakonarson, and D.B. Goldstein. 2010. Rare Variants Create Synthetic Genome-Wide Associations. PLoS Biol. 8(1):e1000294. doi: 10.1371/journal.pbio.1000294.

Endelman, J.B. 2011. Ridge Regression and Other Kernels for Genomic Selection with R Package RrBLUP. Plant Genome J. 4(3):250. doi: 10.3835/plantgenome2011.08.0024.

Endelman, J.B., and J.L. Jannink. 2012. Shrinkage Estimation of the Realized Relationship Matrix. G3 (Bethesda) 2(11):1405-13. doi: 10.1534/g3.112.004259. 
Hanley, J.A., and B.J. McNeil. 1982. The Meaning and Use of the Area under a Receiver Operating Characteristic (ROC) Curve. Radiology 142(1):29-36. doi: 10.1080/02634938208400381.

Huang, X., Y. Zhao, X. Wei, C. Li, A. Wang, Q. Zhao, W. Li, et al. 2012. Genome-Wide Association Study of Flowering Time and Grain Yield Traits in a Worldwide Collection of Rice Germplasm. Nat. Genet. 44(1):32-39. doi: 10.1038/ng.1018.

Jarquin, D., H. Kajiya-Kanegae, C. Taishen, S. Yabe, R. Persa, J. Yu, H. Nakagawa, M. Yamazaki, and H. Iwata. 2019. Coupling Day Length Data and Genomic Prediction Tools for Predicting Time-Related Traits under Complex Scenarios, 703488.

Kang, H.M., J.H. Sul, S.K. Service, N.A. Zaitlen, S.Y. Kong, N.B. Freimer, C. Sabatti, and E. Eskin. 2010. Variance Component Model to Account for Sample Structure in GenomeWide Association Studies. Nat. Genet. 42(4):348-54. doi: 10.1038/ng.548.

Kang, H.M., N.A. Zaitlen, C.M. Wade, A. Kirby, D. Heckerman, M.J. Daly, and E. Eskin. 2008. Efficient Control of Population Structure in Model Organism Association Mapping. Genetics 178(3):1709-23. doi: 10.1534/genetics.107.080101.

Kawahara, Y., M. de la Bastide, J.P. Hamilton, H. Kanamori, W.R. McCombie, S. Ouyang, D.C. Schwartz, et al. 2013. Improvement of the Oryza Sativa Nipponbare Reference Genome Using next Generation Sequence and Optical Map Data 6(1):1-10. doi: 10.1186/1939-84336-1.

Kennedy, B.W., M. Quinton, and J.A. van Arendonk. 1992. Estimation of Effects of Single Genes on Quantitative Traits. J. Anim. Sci. 70(7):2000-2012. doi: 10.1016/j.alcohol.2011.07.002.

Koboldt, D.C., K.M. Steinberg, D.E. Larson, R.K. Wilson, and E.R. Mardis. 2013. The NextGeneration Sequencing Revolution and Its Impact on Genomics. Cell 155(1):27-38. doi: 10.1016/j.cell.2013.09.006.

Korte, A., and A. Farlow. 2013. The Advantages and Limitations of Trait Analysis with GWAS: A Review. Plant Methods 9(1):29. doi: 10.1186/1746-4811-9-29.

Lander, E.S., and D. Botstein. 1989. Mapping Mendelian Factors Underlying Quantitative Traits Using RFLP Linkage Maps. Genetics 121:185-99. http://www.ncbi.nlm.nih.gov/pubmed/2563713.

Li, H. 2012. Exploring Single-Sample Snp and Indel Calling with Whole-Genome de Novo Assembly 28(14):1838-44. doi: 10.1093/bioinformatics/bts280.

Li, J.Y., J. Wang, and R.S. Zeigler. 2014. The 3,000 Rice Genomes Project: New Opportunities and Challenges for Future Rice Research. Gigascience 3(1):1-3. doi: 10.1186/2047-217X$3-8$.

Mansueto, L., R.R. Fuentes, F.N. Borja, J. Detras, J.M. Abrio-Santos, D. Chebotarov, M. Sanciangco, et al. 2017. Rice SNP-Seek Database Update: New SNPs, Indels, and Queries. Nucleic Acids Res. 45(D1):D1075-81. doi: 10.1093/nar/gkw1135.

Mansueto, L., R.R. Fuentes, D. Chebotarov, F.N. Borja, J. Detras, J.M. Abriol-Santos, K. Palis, et al. 2016. SNP-Seek II: A Resource for Allele Mining and Analysis of Big Genomic Data in Oryza Sativa. Curr. Plant Biol. 7-8:16-25. doi: 10.1016/j.cpb.2016.12.003. 
McKenna, A., M. Hanna, E. Banks, A. Sivachenko, K. Cibulskis, A. Kernytsky, K. Garimella, et al. 2009. The Genome Analysis Toolkit: A MapReduce Framework for Analyzing nextGeneration DNA Sequencing Data. Proc. Int. Conf. Intellect. Capital, Knowl. Manag. Organ. Learn. 20:254-60. doi: 10.1101/gr.107524.110.20.

Metzker, M.L. 2010. Sequencing Technologies the next Generation. Nat. Rev. Genet. 11(1):3146. doi: $10.1038 /$ nrg2626.

Misra, G., S. Badoni, R. Anacleto, A. Graner, N. Alexandrov, and N. Sreenivasulu. 2017. Whole Genome Sequencing-Based Association Study to Unravel Genetic Architecture of Cooked Grain Width and Length Traits in Rice. Nat. Sci. Reports 7(1):12478. doi: 10.1038/s41598017-12778-6.

NEI, M. 1973. Analysis of Gene Diversity in Subdivided Populations. Proc. Nat. Acad. Sci. USA 70(12):3321-23. doi: 10.1016/j.jasrep.2018.01.028.

Ott, J., J. Wang, and S.M. Leal. 2015. Genetic Linkage Analysis in the Age of Whole-Genome Sequencing. Nat. Rev. Genet. 16(5):275-84. doi: 10.1038/nrg3908.

Paradis, E., J. Claude, and K. Strimmer. 2004. APE: Analyses of Phylogenetics and Evolution in R Language 20(2):289-90. doi: 10.1093/bioinformatics/btg412.

Saitou, N., and M. Nei. 1987. The Neighbor-Joining Method - a New Method for Reconstructing Phylogenetic Trees. Mol. Biol. Evol. 4(4):406-25.

Storey, J.D., and R. Tibshirani. 2003. Statistical Significance for Genomewide Studies. Proc. Natl. Acad. Sci. 100(16):9440-45. doi: 10.1073/pnas.1530509100.

Stram, D. 2014. Design, Analysis, and Interpretation of Genome-Wide Association Scans. Heidelberg, New York: Springer Science+Business Media.

Wang, W., R. Mauleon, Z. Hu, D. Chebotarov, S. Tai, Z. Wu, M. Li, et al. 2018. Genomic Variation in 3,010 Diverse Accessions of Asian Cultivated Rice. Nature 557(7703):43-49. doi: $10.1038 / \mathrm{s} 41586-018-0063-9$.

Wright, S. 1965. THE INTERPRETATION OF POPULATION STRUCTURE BY FSTATISTICS WITH SPECIAL REGARD TO SYSTEMS OF MATING. Evolution (N. Y). 19:395-420.

Wu, M.C., S. Lee, T. Cai, Y. Li, M. Boehnke, and X. Lin. 2011. Rare-Variant Association Testing for Sequencing Data with the Sequence Kernel Association Test. Am. J. Hum. Genet. 89(1):82-93. doi: 10.1016/j.ajhg.2011.05.029.

Yabe, S., M. Yamasaki, K. Ebana, T. Hayashi, and H. Iwata. 2016. Island-Model Genomic Selection for Long-Term Genetic Improvement of Autogamous Crops. PLoS One 11(4):121. doi: 10.1371/journal.pone.0153945.

Yano, K., E. Yamamoto, K. Aya, H. Takeuchi, P.C. Lo, L. Hu, M. Yamasaki, et al. 2016. Genome-Wide Association Study Using Whole-Genome Sequencing Rapidly Identifies New Genes Influencing Agronomic Traits in Rice. Nat. Genet. 48(8):927-34. doi: 10.1038/ng.3596. 
Yu, J., G. Pressoir, W.H. Briggs, I. Vroh Bi, M. Yamasaki, J.F. Doebley, M.D. McMullen, et al. 2006. A Unified Mixed-Model Method for Association Mapping That Accounts for Multiple Levels of Relatedness. Nat. Genet. 38(2):203-8. doi: 10.1038/ng1702.

Zhang, Z., E. Ersoz, C.Q. Lai, R.J. Todhunter, H.K. Tiwari, M.A. Gore, P.J. Bradbury, et al. 2010. Mixed Linear Model Approach Adapted for Genome-Wide Association Studies. Nat. Genet. 42(4):355-60. doi: 10.1038/ng.546.

Zhao, K., C.-W. Tung, G.C. Eizenga, M.H. Wright, M.L. Ali, A.H. Price, G.J. Norton, et al. 2011. Genome-Wide Association Mapping Reveals a Rich Genetic Architecture of Complex Traits in Oryza Sativa. Nat. Commun. 2:467. doi: 10.1038/ncomms 1467.

\section{Fig. 1. Unrooted phylogenetic tree plot for four non-mixture populations.}

Unrooted phylogenetic tree plot for the four non-mixture populations, which consisted of 112 accessions of japonica (A), 100 accessions of indica (B), 100 accessions of temperate japonica (C), and 100 diverse accessions (D) with neighbor-joining method.

Fig. 2. Correct detection rate for QTN1 in each population under ten conditions.

The barplots of CDR of QTN1 in each population under ten conditions: five levels of $F_{S T}$ of QTN1 and two patterns of polymorphisms of QTN. Blue horizontal dashed lines indicate the CDR in the population A for each population. A: japonica, B: indica, C: temperate japonica, D: diverse, E: A+ B, F: A + C, G: $\mathrm{A}+\mathrm{D}$.

Fig. 3. Boxplots of $-\log _{10}(p)$ of each QTN when QTN1 was polymorphic in japonica (A). 
760 Boxplots of $-\log _{10}(p)$ of each QTN for each mixture population and japonica (A) when QTN1

761 was polymorphic in A. These plots are shown divided into four categories according to the $F_{S T}$

762 value for QTN1 (a: low, b: lower-middle, c: middle, d: higher-middle).

763

764 Fig. 4. Relationship between $\boldsymbol{F}_{S T}, \boldsymbol{H}_{e}$, and the detection power of QTN1.

765 The distribution of each marker is plotted thinly with between subpopulation $F_{S T}$ on the

766 horizontal axis and $H_{e}$ of each subpopulation on the vertical axis. The dark X marks on the plot

767 show the SNPs selected as QTN1s in this study. Red and purple marks were detected by GWAS,

768 and green and yellow ones were not detected by GWAS.

Table 1. Number of SNPs and the diversity level of non-mixture and mixture populations.

\begin{tabular}{lllll}
\hline & Population name & Number of accessions & Number of SNPs & Diversity level $\dagger$ \\
\hline A. & Japonica & 112 & 72,110 & 263.095 \\
B. & Indica & 100 & 427,943 & 660.416 \\
C. & Temperate japonica & 100 & 135,665 & 362.649 \\
D. & Diverse & 100 & 647,731 & 798.646 \\
E. & A + B & 212 & 633,507 & 803.064 \\
F. & A + C & 212 & 151,675 & 334.606 \\
G. & A + D & 212 & 684,774 & 859.678
\end{tabular}

$\dagger$ Diversity level is the index to indicate the degree of genetic diversity, which is described in the

772 Materials and Method section. 